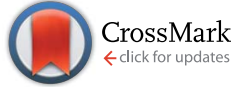

Cite this: RSC Adv., 2015, 5, 13818

\title{
Comprehensive and quantitative analysis for controlling the physical/chemical states and particle properties of nanodiamonds for biological applications $\dagger$
}

\author{
S. Sotoma, ${ }^{a}$ K. Akagi, ${ }^{\text {b }}$ S. Hosokawa, ${ }^{\text {ac }}$ R. Igarashi, ${ }^{a}$ H. Tochio, ${ }^{d}$ Y. Harada ${ }^{e}$ \\ and M. Shirakawa*a
}

\begin{abstract}
The physical/chemical states and properties of nanodiamonds subjected to thermal annealing and air oxidation, which are indispensable processes for the preparation of fluorescent nanodiamonds, were investigated. Specifically, the weight loss, particle size, crystal quality, chemical bonding states of carbon and oxygen, zeta potential, dispersibility, and fluorescent and optically detected magnetic resonance (ODMR) properties were determined using $\mathrm{X}$-ray diffraction (XRD) analysis, transmission electron microscopy (TEM), elemental analysis, dynamic light scattering, Raman analysis, $X$-ray photoelectron spectroscopy (XPS), IR spectroscopy, and a home-made fluorescence and ODMR microscope. The study focused on small-sized nanodiamonds $(\sim 50 \mathrm{~nm})$, which are applicable for biological research. The obtained results should be useful for controlling the mutually-related physical/chemical states and properties of diamond nanoparticles.
\end{abstract}

Received 16th December 2014 Accepted 13th January 2015

DOI: $10.1039 / c 4 r a 16482 b$

www.rsc.org/advances
For the preparation of NDs containing NVCs, thermal annealing and air oxidation processes are indispensable. Thermal annealing at $800{ }^{\circ} \mathrm{C}$ under a vacuum results in the trapping of a moving vacancy by a substitutional nitrogen atom, leading to the formation of an NVC. ${ }^{\mathbf{1 4}}$ Subsequent oxidation in air at 500-600 ${ }^{\circ} \mathrm{C}$ removes the graphite on the ND surface, yielding a fluorescent ND. Many scientists have investigated and reported the optical properties of small-sized NDs $(<50 \mathrm{~nm})$ with potential bio-applications. ${ }^{15,16}$ However, although annealing and oxidation processes affect physical/chemical properties, such as the particle size, crystal quality, and surface chemical termination, little has been learned about the chemical/physical states of NDs.

Gabel et al. reported that air oxidation of NDs is effective for particle size-reduction, ${ }^{17}$ with an average crystal size reduction of $10.6 \mathrm{~nm} \mathrm{~h}^{-1}$ at $600{ }^{\circ} \mathrm{C}$ during air oxidation under atmospheric conditions. Havlik et al. reported that annealing at $900{ }^{\circ} \mathrm{C}$ for one hour resulted in 3-fold brighter ND particles compared to particles annealed under commonly used conditions (temperature and time).${ }^{18}$ In addition, several researchers have reported that high-temperature annealing introduces a graphite on the surface, while oxidation effectively removes this graphite. ${ }^{19,20}$ However, a comprehensive understanding of these physical/chemical states for bio-applicable NDs has not been established. Furthermore, the relationship between these states and ND particle properties, such as dispersibility, fluorescence, and ODMR, has not been reported to date. Changes in these states are expected to affect these important particle properties. $\dagger$ Electronic supplementary information (ESI) available. See DOI: $10.1039 / \mathrm{c} 4 \mathrm{ra} 16482 \mathrm{~b}$ 
By elucidating the relationship between the chemical/physical states and the particle properties, it should be possible to readily prepare fluorescent NDs bearing desired properties. For biological investigations, controlling important factors such as the particle size, crystal quality, surface chemical termination, dispersibility, and fluorescence and ODMR signals will strongly facilitate ND research.

Herein, we report the physical/chemical states and particle properties of pristine NDs and NDs annealed and oxidized at various temperatures and for various lengths of time. The properties were investigated using transmission electron microscopy (TEM), Raman and infrared (IR) spectroscopy, X-ray photoelectron spectroscopy (XPS), X-ray diffraction (XRD) and elemental analyses, and dynamic light scattering. Fluorescence and ODMR signals were detected using a home-built microscope.

\section{Results and discussion}

\subsection{Physical/chemical states of the nanodiamonds}

NDs were annealed at $800{ }^{\circ} \mathrm{C}$ for two hours under a vacuum in order to increase a concentration of NVCs. Subsequently, the NDs were oxidized at $500{ }^{\circ} \mathrm{C}, 525{ }^{\circ} \mathrm{C}, 550{ }^{\circ} \mathrm{C}, 575{ }^{\circ} \mathrm{C}$, or $600{ }^{\circ} \mathrm{C}$ for $1,2,3$, 4, or 5 hours. First, the weight loss of the NDs during annealing and oxidation was investigated. Due to annealing, 7\% of the weight of the NDs was lost. Fig. 1 shows the weight loss of the annealed NDs versus the oxidation time at various oxidation temperatures. The degree of the weight loss highly depended on the oxidation temperature and time. After 5 hours of oxidation, the weight of the NDs remained nearly unchanged at $500{ }^{\circ} \mathrm{C}$, while at $550{ }^{\circ} \mathrm{C}$ and $600{ }^{\circ} \mathrm{C}$, significant losses of approximately $40 \%$ and $99 \%$ were observed, respectively. This weight loss is thought to be due to the isolation of carbon atoms at the surface, which then react with the oxygen and hydrogen in the air to generate carbon oxide. The different degrees of weight loss for the different samples are thought to be related to their physical/chemical states. These three oxidation conditions were thus expected to result in notable differences in the physical/chemical states and were therefore the focus of the present study. Samples are abbreviated as follows: pristine NDs:

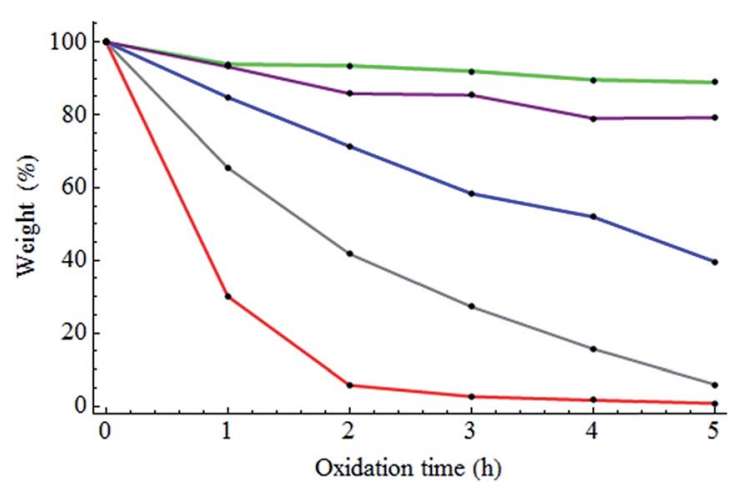

Fig. 1 Weight loss of the NDs versus oxidation time at different oxidation temperatures (green: $500{ }^{\circ} \mathrm{C}$; purple: $525^{\circ} \mathrm{C}$; blue: $550{ }^{\circ} \mathrm{C}$; grey: $575^{\circ} \mathrm{C}$; red: $600^{\circ} \mathrm{C}$ ).
ND-pris; NDs subjected only to thermal annealing at $800{ }^{\circ} \mathrm{C}$ for two hours: ND-800; NDs subjected to thermal annealing at $800{ }^{\circ} \mathrm{C}$ for two hours and subsequently oxidation at 500, 550, or $600{ }^{\circ} \mathrm{C}$ for five hours: ND-500, ND-550, and ND-600, respectively.

2.1.1 Physical state: particle size and crystal quality. Firstly, we confirmed the diamonds crystal structure is maintained for all the prepared samples by means of XRD analysis. Fig. 2 shows XRD spectra of the samples. Each spectrum shows diamond related diffraction peaks at $43.8^{\circ}(111), 75.2^{\circ}(220)$, and $91.4^{\circ}$ (311), indicating each sample maintain the crystal structure. Crystallite size calculated by Scherrer's equation from the (111) peak is shown in Fig. S1 and Table S1. $\dagger$

The ND samples were then observed via TEM in order to elucidate any changes in particle size as a function of the annealing and oxidation processes. Fig. 3 shows photos, TEM images, and size distributions determined from the corresponding TEM images for each of the samples. It can be seen in the photos that ND-pris was a dark-gray color, while ND-800 was black. This change of color may be due to the graphitization of the surface of the NDs during annealing. This graphite was removed by air oxidation, and the degree of whiteness was enhanced with increasing oxidation temperature (a detailed analysis of the surface chemical structure is given in a later section). From the TEM images, it can also be seen that the NDs were not spherical, but had edges. The particle size distributions were similar for ND-pris, ND-800, and ND-500, while the particle sizes for ND-550 and ND-600 were smaller at 35.6 and $14.5 \mathrm{~nm}$, respectively. These results suggested that the surface of ND-800 was graphitized due to annealing, and subsequent oxidation contributed to the removal of the graphite. However, the removal efficiency appeared to depend on the oxidation temperature, because the colors of the different oxidized samples were different. In addition, particle shrinkage occurred when oxidation was performed above $550{ }^{\circ} \mathrm{C}$, and the degree of the size reduction was also temperature dependent.

To obtain further insight into the particle shrinkage and crystal qualities, a Raman spectroscopic analysis was performed. Fig. 4a presents the Raman spectra of the samples. Each spectrum has a D-band ( $\mathrm{sp}^{3}$ carbon), a strain-induced

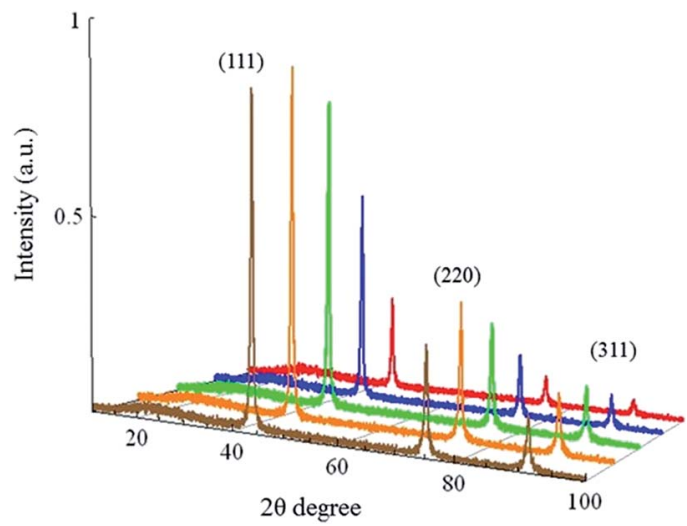

Fig. 2 XRD patterns for ND-pris (brown), ND-800 (orange), ND-500 (green), ND-550 (blue), and ND-600 (red). 
ND-Pris
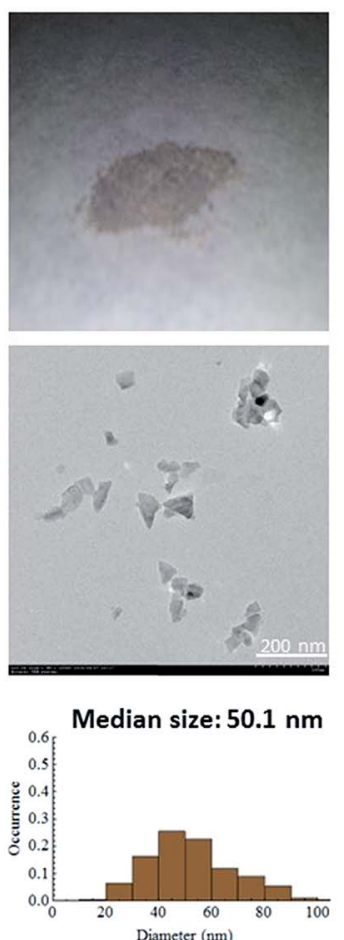

ND-800
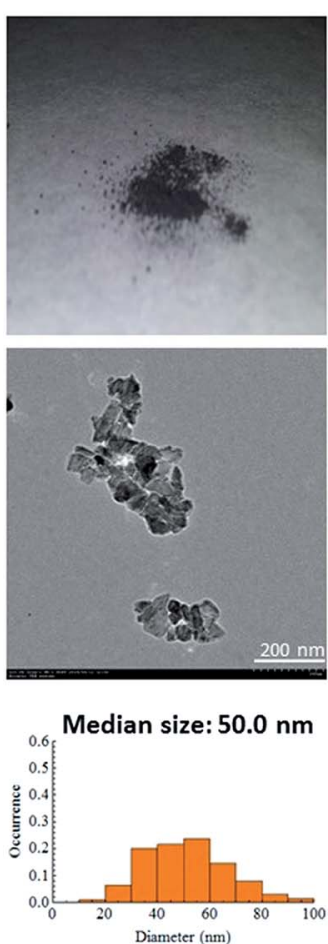

ND-500
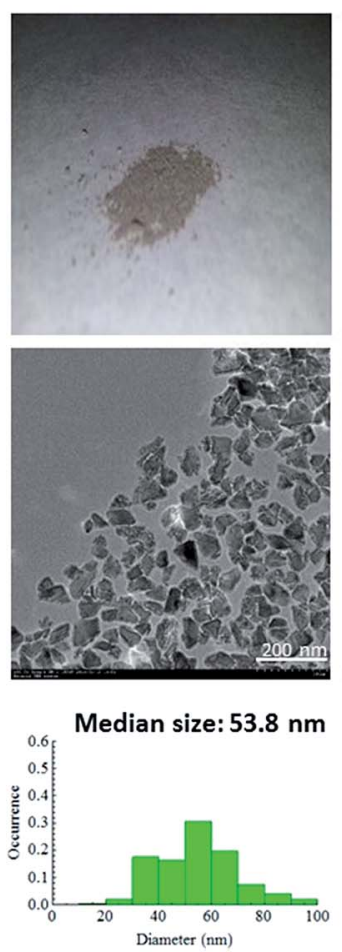

ND-550
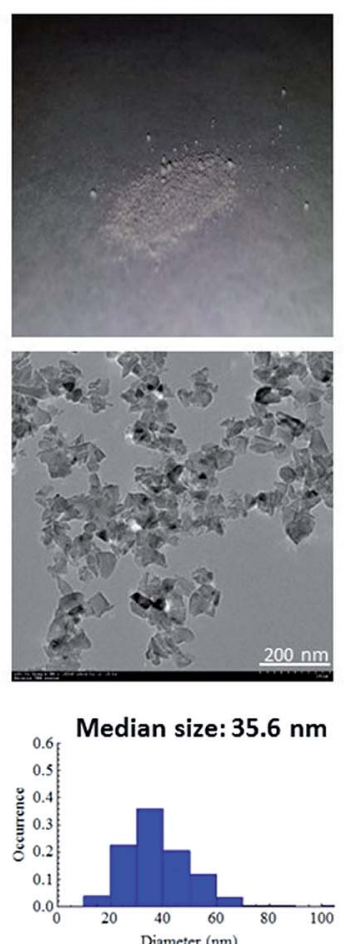

ND-600
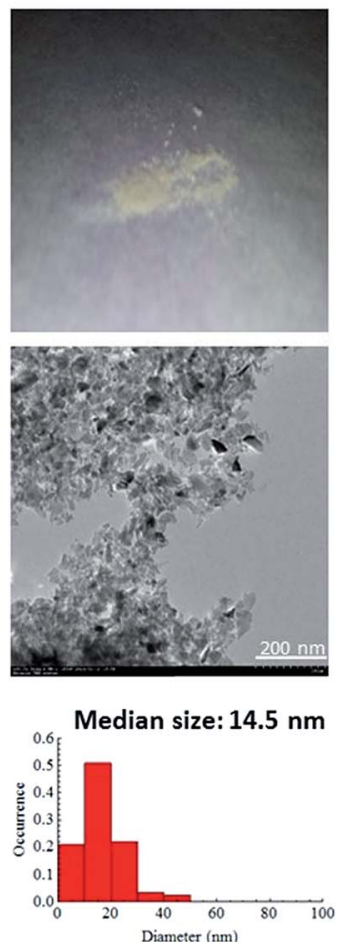

Fig. 3 Photos (upper line), TEM images (middle line), and particle size distributions determined from the corresponding TEM images (lower line) of the ND samples.

band, and a G-band ( $\mathrm{sp}^{2}$ carbon) near 1332, 1450, and $1550 \mathrm{~cm}^{-1}$, respectively. ND-pris exhibited a sharp D-band peak, but the signal for the strain-induced band and the G-band were weak. In contrast, the D-band in the spectrum of ND-800 was drastically enlarged. This result indicated that graphitization of the NDs occurred during annealing, which is supported by the change in color from gray to black (Fig. 3). Because this graphite was readily removed by oxidation at $500{ }^{\circ} \mathrm{C}$, it is thought that it covered only the surfaces of the NDs. Additional notable changes in the Raman spectra for ND-550 and ND-600 were also observed. The G-band was reduced in the spectra of both ND-550 and ND-600, as was the case for ND-500; on the other hand, the strain-induced bands in the spectra of the former samples were largely enhanced. These results indicated that oxidation above $550{ }^{\circ} \mathrm{C}$ induced distortion of the crystal structure, resulting in an increase in the intensity of the straininduced bands. Such distortion was likely caused by the annihilation of carbon atoms at the surface. Fig. $4 \mathrm{~b}$ shows a contour map of the ratio of the Raman signal intensity for diamond divided by that of the strain-induced band for each of the (a)

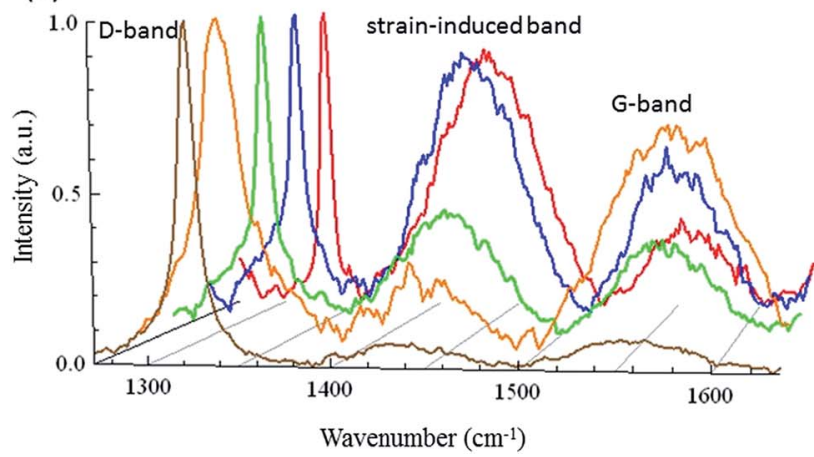

(b)

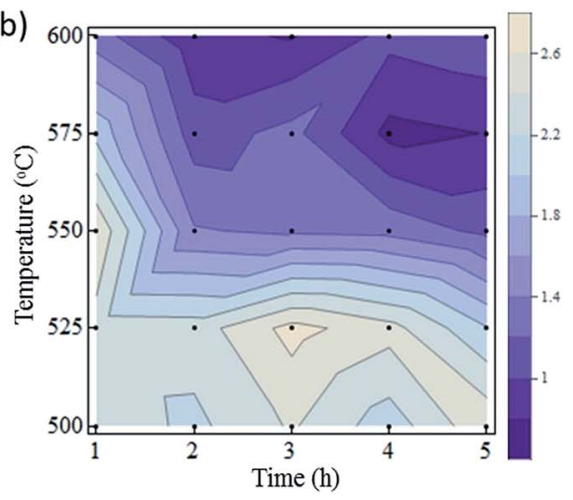

Fig. 4 (a) Raman spectra for ND-pris (brown), ND-800 (orange), ND-500 (green), ND-550 (blue), and ND-600 (red). D-bands (sp 3 carbon), strain-induced bands, and G-bands ( $\mathrm{sp}^{2}$ carbon) near 1332, 1450, and $1550 \mathrm{~cm}^{-1}$, respectively, can be observed in the spectra. (b) Ratios of the Raman diamond peak signal intensities divided by the corresponding amorphous carbon peak intensities for all of the prepared samples as a function of the oxidation time and temperature. 
prepared samples. The black points represent the individual oxidation conditions. It can be seen from the figure that crystal destruction was triggered in the oxidation temperature range from $525{ }^{\circ} \mathrm{C}$ to $550{ }^{\circ} \mathrm{C}$ and appeared to be more temperaturedependent than time-dependent.

To summarize, it was revealed that annealing at $800{ }^{\circ} \mathrm{C}$ introduced graphite on the surfaces of the NDs. Air oxidation at $500{ }^{\circ} \mathrm{C}$ was effective for removal of the introduced surface graphite, but not for changing the particle size. On the other hand, air oxidation above $550{ }^{\circ} \mathrm{C}$ led to both removal of the graphite and particle size reduction, and the degree of each effect increased with increasing oxidation temperature.

2.1.2 Chemical state: bonding state of carbon. Next, the types and numbers of atoms contained in the samples were investigated. Table 1 shows the elemental analysis results for the samples. All of the NDs contained carbon as the major component, along with minor amounts of oxygen and hydrogen and negligible amounts of nitrogen. The results also indicated that the annealing process reduced the number of oxygen atoms below the detection limits for elemental analysis. However, the desorbed oxygen atoms were recovered following oxidation above $500{ }^{\circ} \mathrm{C}$. These results indicated that nearly all of the oxygen atoms existed on the surfaces of the NDs, which was in agreement with the Raman data. In addition, the oxygen to carbon atomic ratios increased with increasing temperature. To learn in more detail about the concentration of the different elements at the surfaces of the NDs, XPS spectra were obtained.

XPS is a favorable method for characterizing material surfaces because the escape depth of an electron is localized to within a few nanometers of the target surface. The survey XPS spectra are shown in Fig. 5a. Each spectrum exhibited C1s and O1s peaks at approximately $285 \mathrm{eV}$ and $533 \mathrm{eV}$, respectively, but their intensities were different for the different samples. The atomic concentrations of carbon and oxygen were calculated from the corresponding intensities of the C1s and O1s peaks. Notably, $13 \%$ of the carbon atoms on the surface of ND-pris were oxidized, which dropped to $8 \%$ for $\mathrm{ND}-800$ and then recovered to $12 \%$ for ND-500. ND-600 had a relatively high oxygen to carbon ratio of $32 \%$, which can be attributed to the high surface-area-to-volume ratio induced by size reduction. A plot of the ratios of oxygen to carbon estimated from the elemental analysis and XPS results is displayed in Fig. S2. $\dagger$ It can be seen in the figure that the XPS and elemental analysis results were similar, although the XPS data were larger than the elemental analysis data. This difference occurred because XPS only measures the surface area of a particle, while elemental analysis evaluates the entire ND particle. It should also be noted

Table 1 Elemental analysis results for the ND samples

\begin{tabular}{lllll}
\hline Element & $\mathrm{H}$ & $\mathrm{C}$ & $\mathrm{N}$ & $\mathrm{O}$ \\
\hline ND-pris & 0.22 & 96.28 & 0.1 & 2.45 \\
ND-800 & 0.05 & 98.89 & 0.37 & 0 \\
ND-500 & 0.24 & 96.06 & 0.1 & 2.03 \\
ND-550 & 0.06 & 94.94 & 0 & 3.04 \\
ND-600 & 0.26 & 76.51 & 0 & 2.88
\end{tabular}

that even for the most oxidized ND sample (ND-600), the oxygen accounted for only $32 \%$ of the total atoms on the surface. This low concentration of oxygen limits the surface functionalization of the NDs.

Fig. 5b presents the deconvoluted high resolution C1s spectra of the samples along with a comparison plot of each component for all of the samples. Each spectrum was calibrated against the $374 \mathrm{eV} \mathrm{Ag3d} \mathrm{peak} \mathrm{as} \mathrm{an} \mathrm{internal} \mathrm{standard} \mathrm{(survey}$ spectra are shown in Fig. S3 $\dagger$ ). The C1s spectra were curve-fitted into three peak components with binding energies of $284 \pm 0.5$, $285 \pm 0.5$, and $286 \pm 0.5 \mathrm{eV}$, which were attributed to $\mathrm{sp}^{2}, \mathrm{sp}^{3}$, and $\mathrm{C}-\mathrm{O}$ bonds. Each of the peak components was also fitted using the Lorenz model, and the existing ratios were estimated. Table 2 shows the probability for each component in the different ND samples. The major component for ND-pris, ND-500, and ND-550 was $\mathrm{sp}^{3}$ bonding, while those for ND-800 and ND-600 were $\mathrm{sp}^{2}$ bonding and $\mathrm{C}-\mathrm{O}$ groups, respectively. As mentioned above, the annealing process introduced graphite, which was then removed during oxidation. Oxygencontaining groups were simultaneously introduced, and the graphite-removal/oxygen-introduction efficiency appeared to be temperature dependent.

The ratios of the $\mathrm{sp}^{3}$ to $\mathrm{sp}^{2}$ carbons in the different ND samples estimated from their Raman and XPS spectra were then compared. Because Raman spectroscopy provides information about the carbon bonding state, the ratios of the Raman signal intensities for the $\mathrm{sp}^{3}$ and strain-induced bands divided by that of the $\mathrm{sp}^{2}$ band were calculated for quantitative comparison of the carbon bonding states in the different samples. Values of 4.1, 1.4, 3.1, 3.8, and 9.5 were obtained for ND-pris, ND-800, ND-500, ND-550, and ND-600, respectively. A plot of the ratios of the $\mathrm{sp}^{3}$ to $\mathrm{sp}^{2}$ bonded carbons in the ND samples estimated from Raman and XPS data is shown in Fig. S4. $\uparrow$ In both cases, the ratio decreased following annealing and increased as the oxidation temperature increased. The differences in the XPS and Raman plots can be attributed to the differences in the observation depths of these analytical techniques.

2.1.3 Chemical state: bonding state of oxygen. Given the experimental results described above, it was anticipated that oxygen species reacted with the carbon in the graphite removed from the ND surface to form alcohol, carboxyl, lactone, and/or epoxy groups. To determine which types of oxygen-containing species were formed in the oxidized samples, FT-IR, and zeta potential analyses were performed. FT-IR spectra of the ND samples are shown in Fig. 6a. A stretch vibration of $\mathrm{O}-\mathrm{H}$ around $3400 \mathrm{~cm}^{-1}$; adsorbed water derived band around $3200 \mathrm{~cm}^{-1}$; aliphatic $\mathrm{C}-\mathrm{H}$ stretching vibrations at 2835-2849 $\mathrm{cm}^{-1}$; carbonyl $\mathrm{C}=\mathrm{O}$ peak at $1750-1778 \mathrm{~cm}^{-1} ; \mathrm{C}=\mathrm{C}$ and/or adsorbed waters peak at $1631 \mathrm{~cm}^{-1} ; \mathrm{COO}^{-}$peak at $1401-1468 \mathrm{~cm}^{-1} ; \mathrm{C}-\mathrm{O}$ peak at $1263-1310 \mathrm{~cm}^{-1}$; C-O-C stretching peak at $1091 \mathrm{~cm}^{-1}$; and $\mathrm{C}-\mathrm{H}$ bending peak at $814-861 \mathrm{~cm}^{-1}$ are observed in the samples. ${ }^{21,22}$ Interestingly, significant differences in the FTIR spectra of ND-500 and ND-550 were not observed, while notable changes were seen in the spectrum of ND-600. Fig. 6b shows the spectra in the range $1000-2000 \mathrm{~cm}^{-1}$. The relative intensity of the $\mathrm{C}-\mathrm{O}$ at $1310 \mathrm{~cm}^{-1}$ and $\mathrm{COO}^{-}$at $1401 \mathrm{~cm}^{-1}$ are diminished, 
(a)
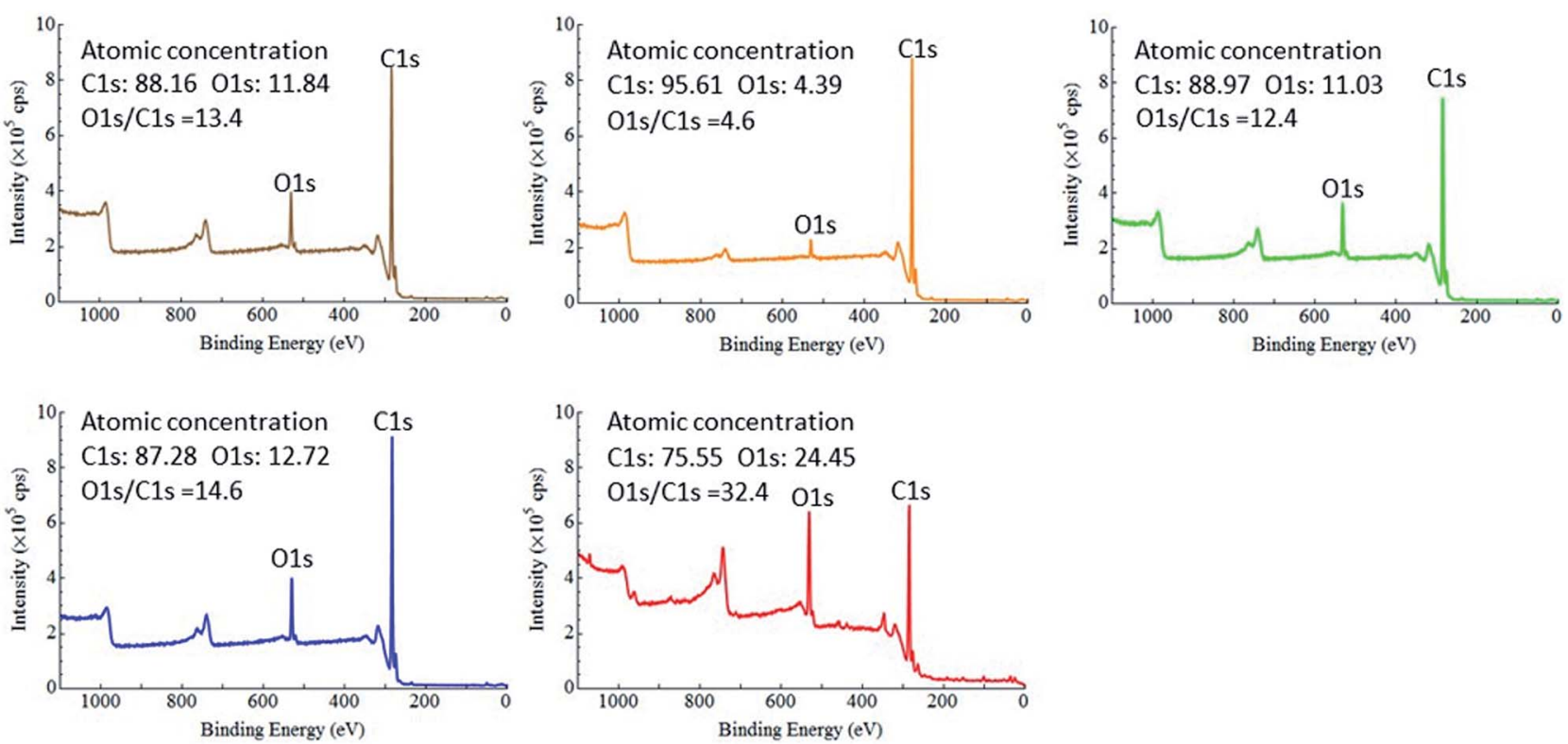

(b)
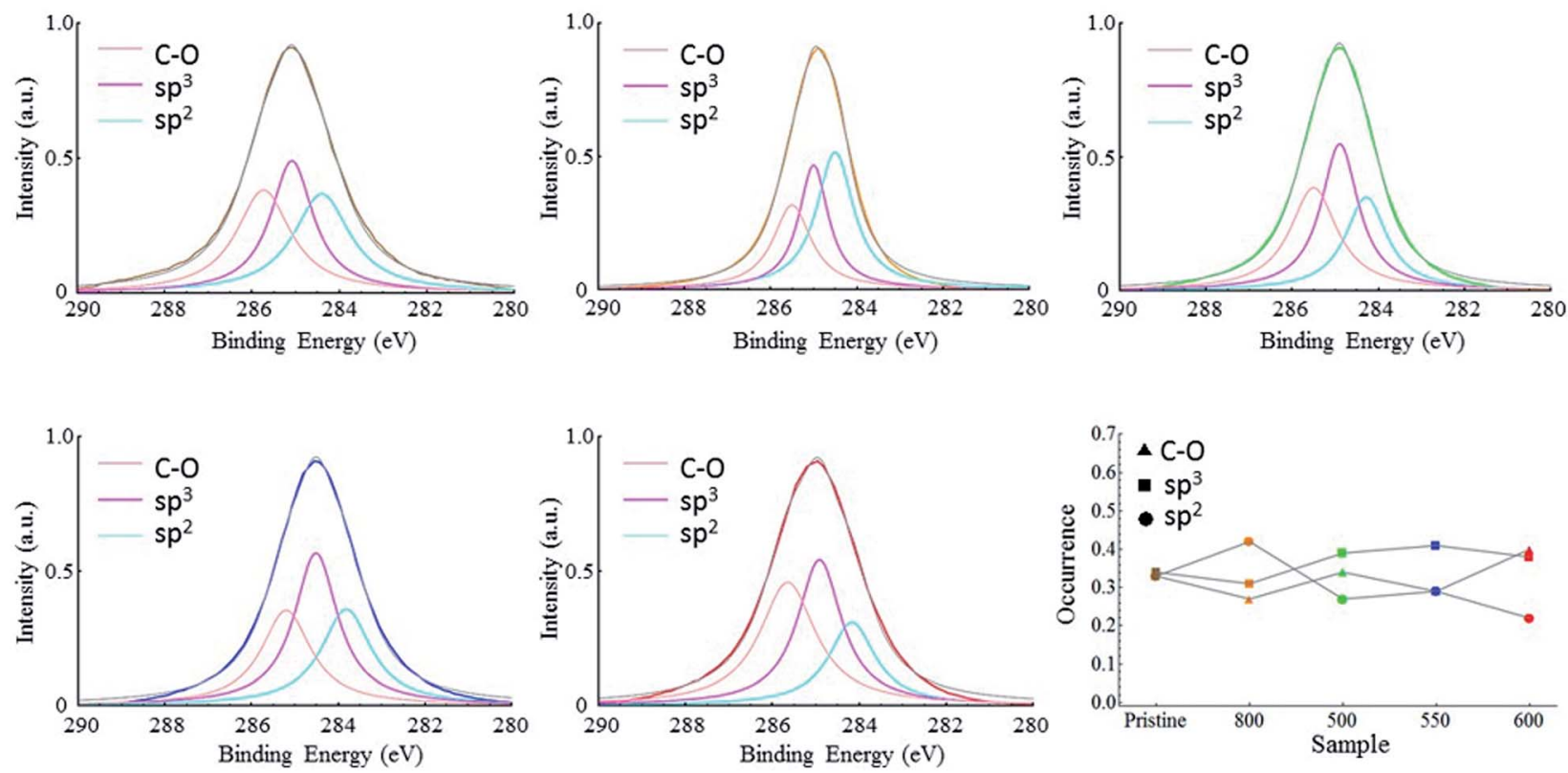

Fig. 5 (a) Survey XPS spectra for ND-pris (brown), ND-800 (orange), ND-500 (green), ND-550 (blue), and ND-600 (red). The atomic concentrations were calculated using the peak intensities for the C1s and O1s orbitals. (b) Deconvoluted high-resolution C1s spectra for ND-pris (brown), ND-800 (orange), ND-500 (green), ND-550 (blue), and ND-600 (red), along with a comparison plot for the components in the different samples.

Table 2 Existence probabilities for the $\mathrm{sp}^{2}, \mathrm{sp}^{3}$, and CO components estimated from Fig. $4 b$

\begin{tabular}{llll}
\hline Component & $\mathrm{sp}^{2}$ & $\mathrm{sp}^{3}$ & $\mathrm{CO}$ \\
\hline ND-pris & 0.33 & 0.34 & 0.33 \\
ND-800 & 0.42 & 0.31 & 0.27 \\
ND-500 & 0.27 & 0.39 & 0.34 \\
ND-550 & 0.29 & 0.41 & 0.29 \\
ND-600 & 0.22 & 0.38 & 0.40
\end{tabular}

while $\mathrm{C}-\mathrm{O}$ at $1332 \mathrm{~cm}^{-1}$ are emerged. We assumed that these spectral changes are attributed to the changes of the oxygen containing groups, possibly due to the conversion from carboxyl groups to a different species such as carboxylic anhydrides. To confirm this, ND-600 was then treated with an aqueous $\mathrm{NaOH}$ solution in order to facilitate the hydration of the surface anhydride groups and thus the formation of free $\mathrm{COOH}$ groups. Gray spectrum in Fig. 6a and b show the NaOH treated ND-600. 
(a)

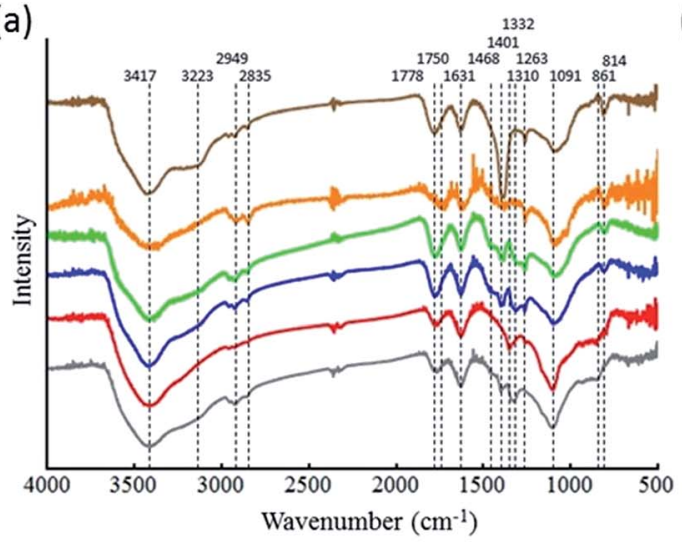

(b)

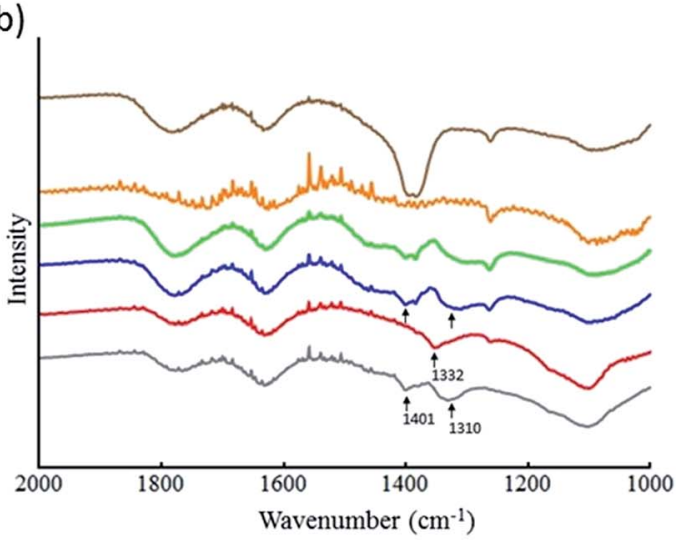

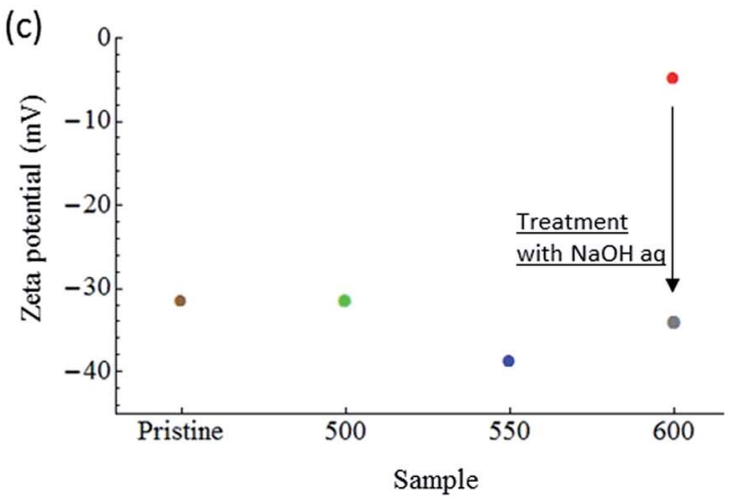

Fig. 6 (a) FT-IR spectra of ND-pris (brown), ND-800 (orange), ND-500 (green), ND-550 (blue), ND-600 (red), and NaOH aq. treated ND-600 (gray). (b) In the spectral range $1000-2000 \mathrm{~cm}^{-1}$. (c) Zeta potentials of the prepared samples and a sample treated in an $\mathrm{NaOH}$ aqueous solution (gray dot).

It is observed that the disappeared peaks at 1310 and $1401 \mathrm{~cm}^{-1}$ are regenerated, and the emerged peaks at $1332 \mathrm{~cm}^{-1}$ disappeared. This data supported the conclusion that the surface $\mathrm{COOH}$ groups were dehydrated in the ND-600. To gain further evidence for this dehydration, the zeta potentials of the ND samples were also determined.

Fig. $6 \mathrm{c}$ shows plots of the zeta potentials for the samples. Note that it was not possible to disperse ND-800 in water, and thus no data was obtained for this sample (Fig. S5†). The zeta potentials were determined to be $-31.5,-31.5,-38.8$, and $-4.9 \mathrm{mV}$ for ND-pris, ND-500, ND-550, and ND-600, respectively. The negative charges are attributed to the presence of $\mathrm{COOH}$ groups on the surfaces of the NDs. The very small zeta potential value for ND-600 indicated that dehydration of the $\mathrm{COOH}$ groups occurred, leading to the formation of carboxylic anhydrides. After this treatment, the zeta potential was found to be $-34.2 \mathrm{mV}$ (gray point in Fig. 6c). This result indicated the regeneration of $\mathrm{COOH}$ groups. Based on these results, it was concluded that excess oxidation led to size reduction accompanied by the introduction of carboxylic anhydride groups on the surfaces of the NDs, which in turn had a lower zeta potential. We further acquired deconvoluted high-resolution O1s XPS spectra of the ND samples, and the results are given in Fig. S6 and Table S2. $\dagger$ The data support the results of IR and zeta potential.
Thus, the overall changes in the physical/chemical states of NDs due to annealing and oxidation have been revealed, and a schematic illustration of the results is presented in Fig. 7.

\subsection{Particle properties}

2.2.1 Dispersibility. The colloidal stability of nanoparticles is well described using Derjaguin and Landau, Verwey and Overbeek (DLVO) theory. ${ }^{23,24}$ This theory treats the interactions among particles in terms of a balance between attractive van der Waals forces and repulsive electrical double layer forces. According to this theory, the size and zeta potential of particles, as well as the ionic strength of a solution of the particles, are important factors for determining their dispersibility. Therefore, the dispersibility of the ND samples was investigated from both theoretical and experimental points of view. Fig. 8 shows a plot of the interaction forces calculated using DLVO theory and experimental data for the time-dependent changes in the intensities of dynamic light scattering for the NDs in saltcontaining aqueous solutions. Photos of their dispersion states are presented in Fig. S7. $\dagger$ The DLVO plots are given in units of $k T$ versus the separation distance between particle surfaces. In a $1 \mathrm{mM}$ salt-containing solution, all of the samples except for ND-600 exhibited a positive local potential energy maximum at a surface distance of approximately $2 \mathrm{~nm}$ (plots of 


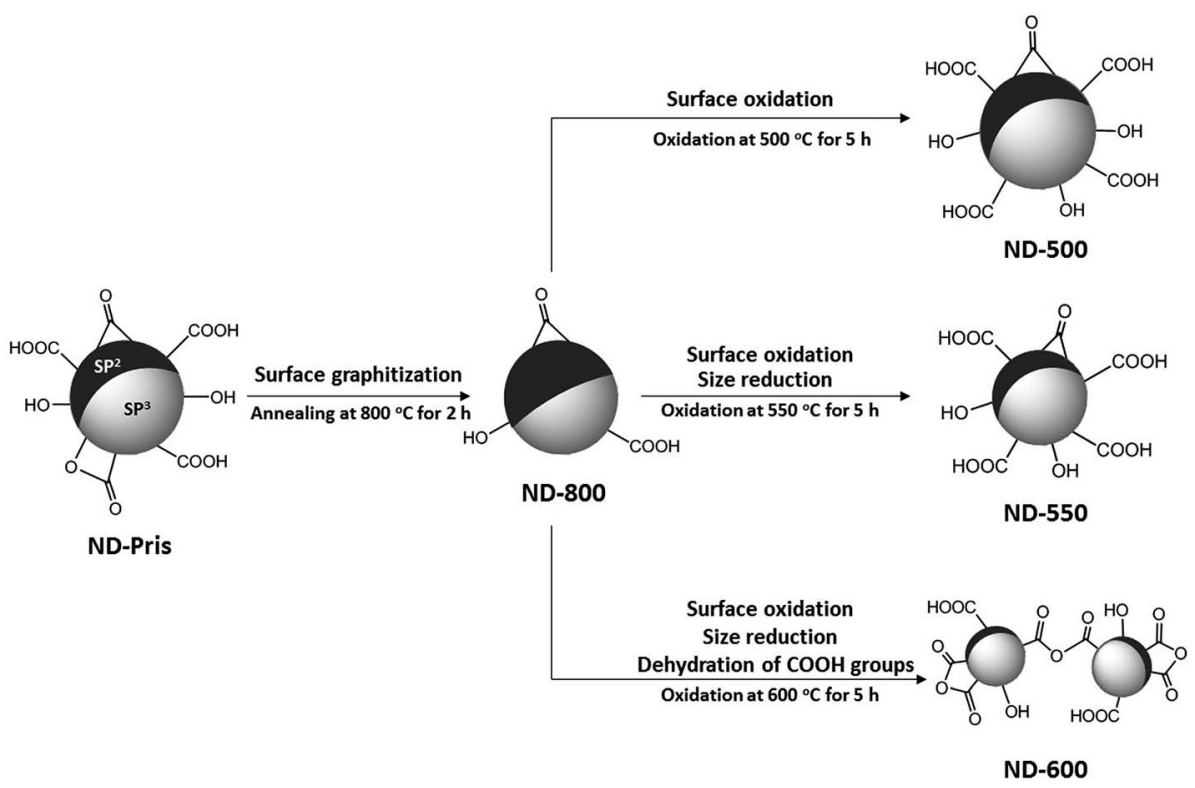

Fig. 7 Schematic illustration of the changes in the physical/chemical states of NDs due to annealing and oxidation.

the surface distance versus solid fraction for the ND samples are shown in Fig. S8 $\dagger$ ). These samples may exhibit good dispersibility because the energy beyond this maximum is required for aggregation. On the other hand, ND-600 would be expected to readily aggregate, because the potential energy of the encountered particles dropped to a primary minimum. Notably,
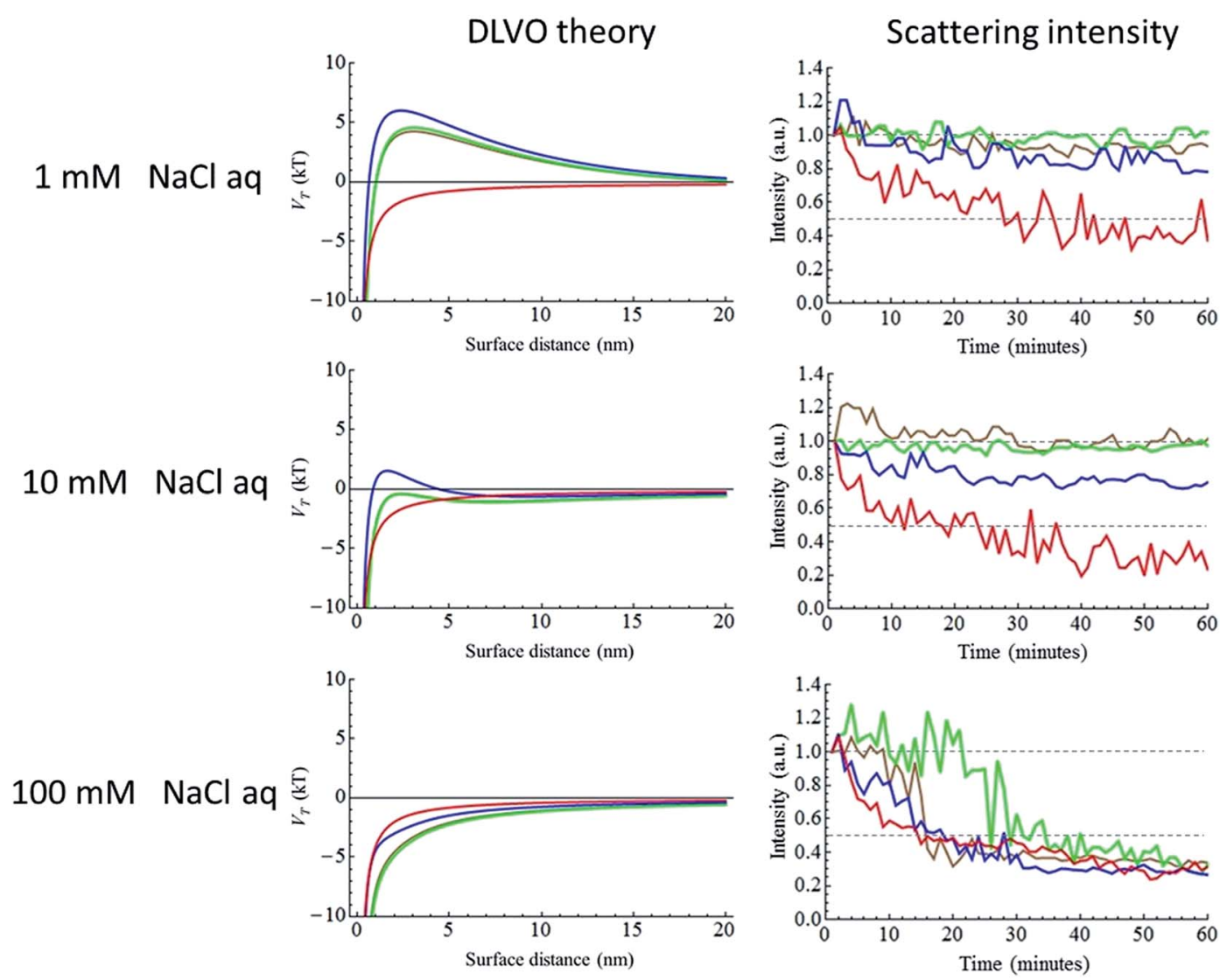

Fig. 8 Plots of the interaction forces calculated using DLVO theory and experimental data for the time-dependent changes in the dynamic light scattering intensity in 1, 10, and $100 \mathrm{mM}$ salt-containing aqueous solutions of NDs. ND-pris, ND-500, ND-550, and ND-600 are represented by brown, green, blue, and red, respectively. 
the experimental data was in agreement with the theoretical results. ND-pris, ND-500, and ND-550 exhibited constant scattering intensities, even after 60 minutes, indicating good dispersibility in solution. In contrast, ND-600 displayed a lower dispersibility in three different salt solutions $(1 \mathrm{mM}, 10 \mathrm{mM}$, and $100 \mathrm{mM}$ ). In addition, for the calculations in a $10 \mathrm{mM}$ salt solution, ND-pris, ND-500, and ND-550 each exhibited a local maximum near zero, and thus the samples were assumed to be rapidly dispersed and then gradually aggregated over an extended period. The experimental data also supported this result; the samples did not precipitate in as short a period. For the $100 \mathrm{mM}$ solutions, the samples were predicted to precipitate immediately, and this result confirmed experimentally. Few literature reports mention the dispersibility of NDs in terms of DLVO theory. These results demonstrated that this theory provides valid information on the dispersion state of NDs. Furthermore, it can be concluded from the results that in order to maintain dispersion in high-concentration salt-containing solutions, chemical modification of the surface is required. Dendrimer and lipid coatings have been reported to enhance the dispersibility of NDs. ${ }^{25,26}$ We further measured particle size of dispersible ND samples by means of DLS (Fig. S9†). The results are in good accordance with the size estimated from TEM images.

2.2.2 Fluorescence and ODMR properties. Next, the fluorescence and ODMR spectra of the ND samples were evaluated. There are two types of nitrogen-vacancy centers in NDs, each with a different charged state. One is neutral $\left(\mathrm{NV}^{0}\right)$, while the other is negative $\left(\mathrm{NV}^{-}\right)$, and they appear as zero-phonon lines (ZPLs) at $575 \mathrm{~nm}$ and $637 \mathrm{~nm}$, respectively. ${ }^{27}$ Both NVCs emit fluorescence, but only $\mathrm{NV}^{-}$can be detected using ODMR. ${ }^{28,29}$ The ground state of $\mathrm{NV}^{-}$is a spin-triplet, and the spin sublevels $m_{\mathrm{s}}=0$ and $m_{\mathrm{s}}= \pm 1$ are split by $2870 \mathrm{MHz}$. In addition, the spin sublevel $m_{\mathrm{s}}= \pm 1$ is degenerate in the absence of an external magnetic field. Microwave (MW) irradiation at this frequency induces electron spin magnetic resonance (ESR) between $m_{\mathrm{s}}=0$ and the degenerate $m_{\mathrm{s}}= \pm 1$ spin levels, which results in a decrease in the fluorescence intensity from $\mathrm{NV}^{-}$due to spindependent intersystem crossing. ODMR functions via detection of ESR through changes in the fluorescence intensity.
ODMR signals are assumed to be affected by the surface chemical termination, crystal quality, and particle size of NDs. Thus, the fluorescence and ODMR signals for the NDs prepared in this study were investigated.

Fig. 9a shows the fluorescence spectra of the bulk samples. The data for ND-pris is not shown because this sample was not subjected to annealing, and thus no NVCs were introduced into the NDs. ND-800 exhibited a very weak fluorescence intensity, possibly due to absorption of the fluorescence from the NVCs by the surface graphite. The other samples exhibited similar fluorescence spectra, with ZPLs for $\mathrm{NV}^{0}$ and $\mathrm{NV}^{-}$at 575 and $637 \mathrm{~nm}$, respectively. The ODMR spectra of ND-800, ND-500, ND-550, and ND-600 are shown in Fig. 9b. Each sample clearly exhibited MW-dependent reduction of the fluorescence intensity at $2870 \mathrm{MHz}$. Notably, in the spectrum of ND-800, the signal was weaker and broader than that in the spectrum of ND-500, which was likely caused by the graphite. The strongest ODMR signal of 4.7\% was observed for ND-500. The ODMR signal then deceased for ND-550 and ND-600 to $3.6 \%$ and $2.4 \%$, respectively. This decrease in the intensity is assumed to derive from the decrease in the total number of NVCs (both $\mathrm{NV}^{0}$ and $\mathrm{NV}^{-}$) contained in the NDs due to their reduction in size. However, it must be considered that the ODMR signal is determined as shown in eqn (1):

$$
I_{\mathrm{ODMR}}=\left(I_{\mathrm{OFF}}-I_{\mathrm{ON}}\right) / I_{\mathrm{OFF}},
$$

where $I_{\mathrm{ODMR}}, I_{\mathrm{OFF}}$, and $I_{\mathrm{ON}}$ indicate the intensity of the ODMR, and the peak intensities of the distribution when the MW irradiation are off and on, respectively. Thus, the signal may not be influenced by the number of $\mathrm{NVCs}$ if $\mathrm{NV}^{0}$ and $\mathrm{NV}^{-}$randomly exist in the NDs. In addition, the strain induced splitting coefficient values ( $E$-values) of the ND samples were notable. The spin Hamiltonian of an $\mathrm{NV}^{-}$is expressed using eqn (2):

$$
H=D\left[S_{z}{ }^{2}-\frac{1}{3} S(S+1)\right]+E\left(S_{x}{ }^{2}-S_{y}{ }^{2}\right),
$$

where $S, D$, and $E$ are the electron spin vector $(S=1$ for the triplet state), the fine-structure zero-field splitting, and the strain induced splitting coefficient, respectively. Thus, crystal (a)

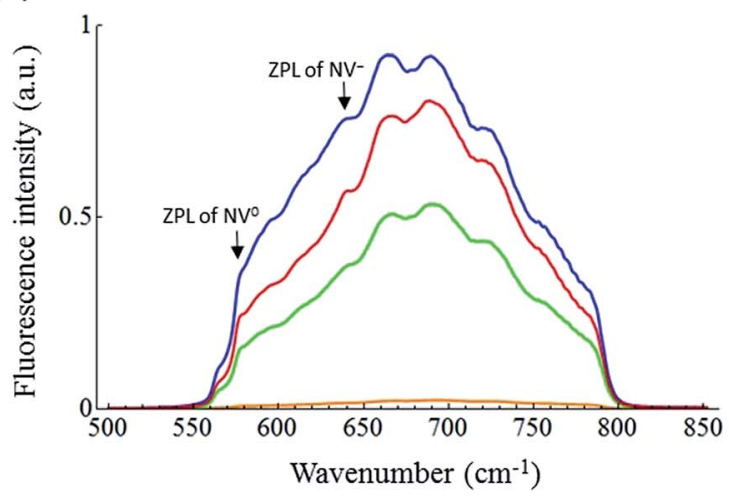

(b)

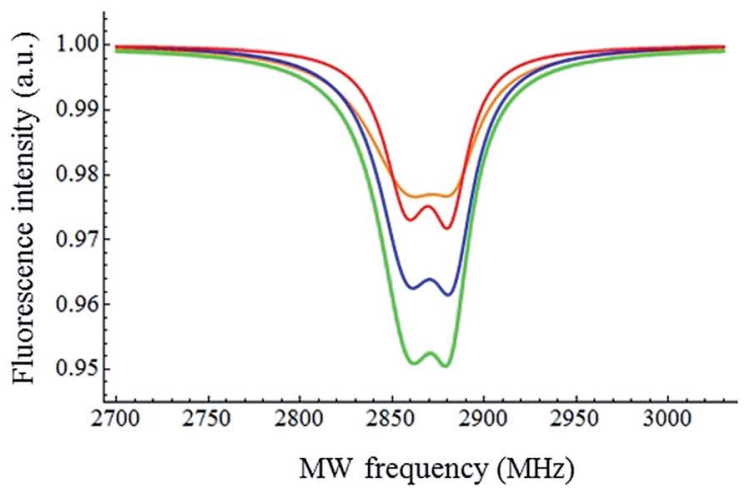

Fig. 9 (a) Fluorescence and (b) ODMR spectra of ND-800 (orange), ND-500 (green), ND-550 (blue), and ND-600 (red). 
Table 3 ODMR signal data for Fig. 8b

\begin{tabular}{llll}
\hline Parameter & Intensity $(\%)$ & HWHM $(\mathrm{MHz})$ & $E$-value $(\mathrm{MHz})$ \\
\hline ND-800 & 2.3 & 16.8 & 13.0 \\
ND-500 & 4.7 & 13.7 & 11.5 \\
ND-550 & 3.6 & 14.5 & 12.3 \\
ND-600 & 2.4 & 12.9 & 11.6 \\
\hline
\end{tabular}

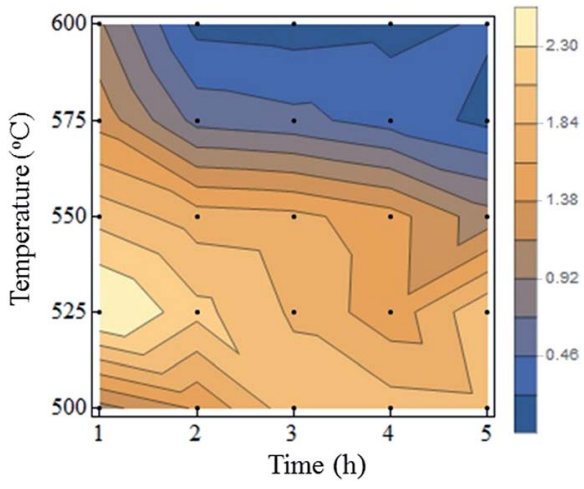

Fig. 10 ODMR intensities for all of the prepared ND samples as a function of the oxidation time and temperature.

distortion near the NVCs is estimated by measuring the $E$-value. Table 3 shows the intensity, half-width at half-maximum (HWHM), and $E$-values for the ODMR spectra of the ND samples. The values were estimated by fitting the ODMR spectra using two Lorenz functions. Unlike for the results of the Raman spectroscopic analysis, no dependence of the crystal distortion on the oxidation process was observed, these results implied that ODMR-active $\mathrm{NV}^{-}$sites that caused distortion of the crystal structure rarely existed close to the surface. In other words, the charge states of the NVCs were converted from $\mathrm{NV}^{-}$to $\mathrm{NV}^{0}$ close to the surfaces of the NDs, resulting in lower-intensity ODMR spectra. It has been reported that surface termination using oxygen or fluorine enhances the $\mathrm{NV}^{-} / \mathrm{NV}^{0}$ ratio. ${ }^{30,31}$ Oxygen or fluorine termination may thus result in restriction of such an undesirable conversion of NVCs. Finally, the ODMR intensities of all of the prepared samples were plotted as a function of the oxidation time and temperature (Fig. 10). A discrete maximum for the ODMR intensity was identified in the samples oxidized at $525{ }^{\circ} \mathrm{C}$ for 1 hour. Oxidation at temperatures above $575{ }^{\circ} \mathrm{C}$ resulted in NDs with a weak ODMR intensity of less than half the maximum value. It is possible, therefore, that particle shrinkage may critically affect the ODMR intensity.

\section{Conclusions}

Changes in the physical/chemical states of NDs due to annealing and oxidation have been quantitatively analyzed, and several key points have been elucidated.

(1) Weight loss in the NDs occurred via several processes.

(2) A surface graphite introduced during annealing was oxidized in air at temperatures above $500{ }^{\circ} \mathrm{C}$.
(3) Distortion of the crystal during oxidation at $525-550{ }^{\circ} \mathrm{C}$ was accompanied by particle shrinkage.

(4) The $\mathrm{sp}^{3} / \mathrm{sp}^{2}$ ratio increased with increasing temperature, and $\mathrm{COOH}$ groups introduced at lower oxidation temperature were dehydrated at higher oxidation temperatures, resulting in NDs with low zeta potentials.

In addition to these results, it was found that the dispersibility, fluorescence, and ODMR properties of the NDs are related to their physical/chemical properties. Notably, there is a strong relationship between the dispersibility of NDs and their physical/chemical states, and the dispersibility can be estimated in advance using DLVO theory. In addition, it was determined that the ODMR signal intensity for NDs may be influenced by its $\mathrm{sp}^{2}$ ratio and particle size; the ODMR signal decreased with decreasing particle size, but crystal distortion around $\mathrm{NV}^{-}$was not observed, indicating that an NVC existing at the surface was converted from $\mathrm{NV}^{-}$to $\mathrm{NV}^{0}$. Furthermore, the optimal oxidation conditions for a discrete maximum ODMR intensity were determined. These experimental results suggest that it should be possible to control the physical/chemical states and properties of NDs through adjustment of the annealing and oxidation processes, and will thus open avenues for facilitating their bio-application.

\section{Experimental}

NDs (MD50) synthesized by a static high-pressure hightemperature (HPHT) method were purchased from Tomei Diamond Co. Ltd., and used in this study. Ion irradiation, which is the common method for creating additional vacancies in NDs, ${ }^{27}$ was not applied to the materials. TEM images were obtained using a Hitachi H-7650. Raman spectra were obtained using a Horiba XploRa in the range from 1200 to $1650 \mathrm{~cm}^{-1}$ in order to evaluate the distribution of the diamond $\mathrm{sp}^{3}$ band $\left(1332 \mathrm{~cm}^{-1}\right)$, the strain-induced band $\left(1450 \mathrm{~cm}^{-1}\right)$, and the graphite $\mathrm{sp}^{2}$ band $\left(1550 \mathrm{~cm}^{-1}\right)$. A $488 \mathrm{~nm}$ excitation laser was used. X-ray diffraction patterns were recorded using a power X-ray diffractometer (MultiFlex DR, Rigaku, Tokyo Japan) with a CuK $\alpha$ source (X-ray wavelength $0.15405 \mathrm{~nm}$ ) and an acceleration voltage and anode current of $30 \mathrm{kV}$ and $30 \mathrm{~mA}$, respectively. XPS spectra were recorded using an ULVAC PHI 5500MT system. Samples mounted on indium foil were analyzed using $\mathrm{MgK} \alpha$ radiation $(15 \mathrm{kV}, 400 \mathrm{~W})$ in a vacuum chamber $\left(<1 \times 10^{-8}\right.$ Torr $)$. Precise measurement and characterization of the energies of the C1s and $01 \mathrm{~s}$ peaks were achieved by referencing them to the $3 \mathrm{~d}$ peak of $\mathrm{Ag}$ at $374 \mathrm{eV}$. FTIR spectra were obtained on a JASCO FT/IR4200 using standard KBr-pellets with a resolution of $2 \mathrm{~cm}^{-1}$. The zeta potentials of the samples in neutral water were measured using a Malvern Zeta sizer Nano instrument. The concentration of each ND sample was $1 \mathrm{mg} \mathrm{ml}^{-1}$ in a saltcontaining aqueous solution, and the samples were sonicated for 5 minutes prior to measurement. Time-dependent changes in the dynamic light scattering intensities of the samples ( $1 \mathrm{mg} \mathrm{ml} \mathrm{m}^{-1}$ in Milli-Q water) were determined using a Wyatt DynaPro NanoStar. Fluorescence and optically detected magnetic resonance spectra were recorded using a home-built microscope established by Igarashi et al. ${ }^{32}$ The field of view 
was illuminated using a $532 \mathrm{~nm} \mathrm{Nd:YAG} \mathrm{laser} \mathrm{at} 7 \mathrm{~mW}$, and fluorescence images $(40 \mu \mathrm{m} \times 40 \mu \mathrm{m})$ were obtained using an electron multiplying charge-coupled device (EMCCD) camera, while fluorescence spectra were recorded using a Hamamatsu photonics PMA-12. The emitted light was collected using an oil immersion $100 \times$ objective lens (numerical aperture $=1.49$ ) and passed through a dichroic mirror centered at $575 \mathrm{~nm}$ and a long (short)-wave pass filter longer than $590 \mathrm{~nm}$ (shorter than 842 $\mathrm{nm}$ ) to detect the intrinsic signal. The microscope was equipped with a computer-controlled moving stage.

Treatment of ND-600 in an $\mathrm{NaOH}$ aqueous solution was performed using the following procedure. $\mathrm{NaOH}(0.1 \mathrm{M})$ was added to the NDs and the suspension was stirred for two hours at $90{ }^{\circ} \mathrm{C}$. After washing three times with Milli-Q water, the NDs were stirred in $0.1 \mathrm{M} \mathrm{HCl}$ for two hours at $90{ }^{\circ} \mathrm{C}$. The NDs were then centrifuged and washed three times with Milli-Q water.

\section{Acknowledgements}

The authors thank Prof. Tsunehiro Tanaka, Graduate School of Engineering, Kyoto University, for help with the XRD and XPS analyses; Mr Yuta Kumiya, Graduate School of Engineering, Kyoto University, for help with the fluorescence measurements; and Mr Erik Walinda, Graduate School of Engineering, Kyoto University, for his scientific discussions. This research was supported by the World Premier International Research Center World Researchers (NEXT Program). Initiative (WPI), Ministry of Education, Culture, Sports, Science and Technology (MEXT) Japan, the Japan Science and Technology Agency under the Core Research for Evolutional Science (CREST), and the Japan Society for the Promotion of Science under the Funding Program for Next-Generation.

\section{Notes and references}

1 I. Aharonovich, A. Greentree and S. Prawer, Nat. Photonics, 2011, 5, 397-405.

2 S.-J. Yu, M.-W. Kang, H.-C. Chang, K.-M. Chen and Y.-C. Yu, J. Am. Chem. Soc., 2005, 127, 17604-17605.

3 G. Liaugaudas, G. Davies, K. Suhling, R. U. A. Khan and D. J. F. Evans, J. Phys.: Condens. Matter, 2012, 24, 435503.

4 F. Jelezko and J. Wrachtrup, Phys. Status Solidi, 2006, 203, 3207-3225.

5 A. Krueger and D. Lang, Adv. Funct. Mater., 2012, 22, 890-906. 6 A. M. Schrand, H. Huang, C. Carlson, J. J. Schlager, E. Omacr Sawa, S. M. Hussain and L. Dai, J. Phys. Chem. B, 2007, 111, 2-7.

7 Y. Y. Hui, C.-L. Cheng and H.-C. Chang, J. Phys. D: Appl. Phys., 2010, 43, 374021.

8 J. M. Say, C. Vreden, D. J. Reilly, L. J. Brown, J. R. Rabeau and N. J. C. King, Biophys. Rev., 2011, 3, 171-184.

9 H. Bernien, B. Hensen, W. Pfaff, G. Koolstra, M. S. Blok, L. Robledo, T. H. Taminiau, M. Markham, D. J. Twitchen, L. Childress and R. Hanson, Nature, 2013, 497, 86-90.

10 T. Staudacher, F. Shi, S. Pezzagna, J. Meijer, J. Du, C. A. Meriles, F. Reinhard and J. Wrachtrup, Science, 2013, 339, 561-563.
11 M. Geiselmann, M. L. Juan, J. Renger, J. M. Say, L. J. Brown, F. J. G. de Abajo, F. Koppens and R. Quidant, Nat. Nanotechnol., 2013, 8, 175-179.

12 Y. Y. Hui and H.-C. Chang, J. Chin. Chem. Soc., 2014, 61, 6776.

13 R. Schirhagl, K. Chang, M. Loretz and C. L. Degen, Annu. Rev. Phys. Chem., 2014, 65, 83-105.

14 X. Song, G. Wang, X. Liu, F. Feng and J. Wang, Appl. Phys. Lett., 2013, 102, 133109.

15 A. Schrand, S. a. C. Hens and O. Shenderova, Crit. Rev. Solid State Mater. Sci., 2009, 34, 18-74.

16 J. Tisler, G. Balasubramanian, B. Naydenov, R. Kolesov, B. Grotz, R. Reuter, J.-P. Boudou, P. a. Curmi, M. Sennour, A. Thorel, M. Börsch, K. Aulenbacher, R. Erdmann, P. R. Hemmer, F. Jelezko and J. Wrachtrup, ACS Nano, 2009, 3, 1959-1965.

17 T. Gaebel, C. Bradac, J. Chen, J. M. Say, L. Brown, P. Hemmer and J. R. Rabeau, Diamond Relat. Mater., 2012, 21, 28-32.

18 J. Havlik, V. Petrakova, I. Rehor, V. Petrak, M. Gulka, J. Stursa, J. Kucka, J. Ralis, T. Rendler, S.-Y. Lee, R. Reuter, J. Wrachtrup, M. Ledvina, M. Nesladek and P. Cigler, Nanoscale, 2013, 5, 3208-3211.

19 J. Cebik, J. K. McDonough, F. Peerally, R. Medrano, I. Neitzel, Y. Gogotsi and S. Osswald, Nanotechnology, 2013, 24, 205703.

20 S. Osswald, G. Yushin, V. Mochalin, S. O. Kucheyev and Y. Gogotsi, J. Am. Chem. Soc., 2006, 128, 11635-11642.

21 O. Shenderova, M. Panich, S. Moseenkov, S. C. Hens, V. Kuznetsov and H.-M. Vieth, J. Phys. Chem. C, 2011, 115, 19005-19011.

22 V. Y. Osipov, E. Aleksenskiy, I. Shames, M. Panich, M. S. Shestakov and A. Y. Vul, Diamond Relat. Mater., 2011, 20, 1234-1238.

23 E. Verwey and J. Overbeek, Theory of the Stability of Lyophobic Colloids, Elsevier, 1948.

24 B. V. Deryagyin and L. Landau, Acta Phys. Chem., 1941, 15, 633.

25 Y. Hui, B. Zhang and Y. Chang, Opt. Express, 2010, 18, 21352143.

26 L. Zhao, T. Takimoto, M. Ito, N. Kitagawa, T. Kimura and N. Komatsu, Angew. Chem., Int. Ed., 2011, 50, 1388-1392.

27 Y.-R. Chang, H.-Y. Lee, K. Chen, C.-C. Chang, D.-S. Tsai, C.-C. Fu, T.-S. Lim, Y.-K. Tzeng, C.-Y. Fang, C.-C. Han, H.-C. Chang and W. Fann, Nat. Nanotechnol., 2008, 3, 284288.

28 P. Maletinsky, S. Hong, M. S. Grinolds, B. Hausmann, M. D. Lukin, R. L. Walsworth, M. Loncar and A. Yacoby, Nat. Nanotechnol., 2012, 7, 320-324.

29 V. R. Horowitz, B. J. Alemán, D. J. Christle, A. N. Cleland and D. D. Awschalom, Proc. Natl. Acad. Sci. U. S. A., 2012, 109, 13493-13497.

30 K. Fu, C. Santori, P. E. Barclay and R. G. Beausoleil, Appl. Phys. Lett., 2010, 96, 121907.

31 S. Cui and E. L. Hu, Appl. Phys. Lett., 2013, 103, 051603.

32 R. Igarashi, Y. Yoshinari, H. Yokota, T. Sugi, F. Sugihara, K. Ikeda, H. Sumiya, S. Tsuji, I. Mori, H. Tochio, Y. Harada and M. Shirakawa, Nano Lett., 2012, 12, 5726-5732. 